\title{
Performance rating of the transmuted exponential distribution: an analytical approach
}

\author{
Enahoro A. Owoloko ${ }^{1}$, Pelumi E. Oguntunde ${ }^{1^{*}}$ and Adebowale O. Adejumo ${ }^{2}$
}

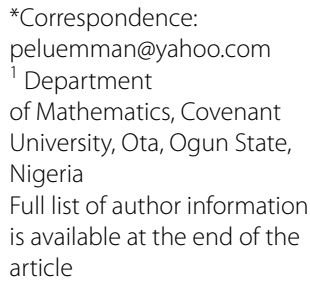

\begin{abstract}
In this article, the so called Transmuted Exponential (TE) distribution was applied to two real life datasets to assess its potential flexibility over some other generalized models. Various statistical properties of the TE distribution were also identified while the method of maximum likelihood estimation was used to estimate the model parameters.
\end{abstract}

Keywords: Estimation, Flexibility, Maximum likelihood estimation, Properties, Transmuted Exponential

\section{Background}

Attempts to generalize the Exponential distribution have led to the developement of Beta Exponential distribution (Nadarajah and Kotz 2006), Kumaraswamy Exponential distribution (Cordeiro and de Castro 2011), Generalized Exponential distribution (Gupta and Kundu 1999, 2007) and Exponentiated Exponential distribution (Gupta 2001). These distributions have been found to be more flexibly than the Exponential distribution when applied to real life data sets.

Let $\mathrm{X}$ denotes a random variable, the probability density function (pdf) and the cumulative density function (cdf) of an Exponential distribution with parameter $\theta$ can be defined using an alternative parameterization as;

$$
g(x)=\frac{1}{\theta} e^{-\left(\frac{x}{\theta}\right)} ; \quad x \geq, \theta>0
$$

and

$$
G(x)=1-\frac{1}{\theta} e^{-\left(\frac{x}{\theta}\right)} ; \quad x \geq 0, \theta>0
$$

respectively.

where; $\theta$ is the scale parameter

Several generalized families of distributions have been proposed in the literature, for instance, the $\beta$-G; (Eugene et al. 2002), Kumaraswamy-G; (Cordeiro and de Castro 2011), Transmuted family of distributions; (Shaw and Buckley 2007), Gamma-G (type 1); (Zografos and Balakrishnan 2009), McDonald-G; (Alexander et al. 2012), GammaG (type 2); (Ristic et al. 2012), Gamma-G (type 3); (Torabi and Montazari 2012),

(c) 2015 Owoloko et al. This article is distributed under the terms of the Creative Commons Attribution 4.0 International License (http://creativecommons.org/licenses/by/4.0/), which permits unrestricted use, distribution, and reproduction in any medium, provided you give appropriate credit to the original author(s) and the source, provide a link to the Creative Commons license, and indicate if changes were made. 
Log-gamma-G; Amini et al. (2012), Exponentiated T-X; Alzaghal et al. (2013), Exponentiated-G (EG); (Cordeiro et al. 2013), Logistic-G; Torabi and Montazari (2014), GammaX; (Alzaatreh et al. 2013), Logistic-X; (Tahir et al. 2015), Weibull-X; (Alzaatreh et al. 2013), Weibull-G; (Bourguignon et al. 2014) and Beta Marshall-Olkin family of distributions; (Alizadeh et al. 2015) and many others are available in the literature.

Of interest to us in this article is the Transmuted family of distribution which was obtained using the quadratic rank transmutation map. The transmuted family of distributions has been adopted by several notable authors to generalize known theoretical models, the Transmuted Weibull distribution; Aryal and Tsokos (2011), Transmuted Rayleigh distribution; (Merovci 2013), Transmuted Exponentiated Modified Weibull distribution; (Ashour and Eltehiwy 2013a), Transmuted Modified Weibull distribution; Khan and King (2013), Transmuted Lomax distribution; (Ashour and Eltehiwy 2013b), Transmuted Exponentiated Gamma distribution; Hussian (2014), Transmuted Inverse Rayleigh distribution; Ahmad et al. (2014), Transmuted Pareto distribution; (Merovci and Puka 2014), Transmuted Inverse Weibull distribution; (Khan et al. 2014), Transmuted Modified Inverse Weibull Distribution; (Elbatal 2013), Transmuted Additive Weibull distribution; (Elbatal and Aryal 2013), Transmuted Complementary Weibull Geometric Distribution; (Afify et al. 2014), Transmuted Inverse Exponential distribution; (Oguntunde and Adejumo 2015), Transmuted Size-Biased Exponential distribution; Ahmad et al. (2015) and Transmuted Gompertz distribution; (Abdul-Moniem and Seham 2015); are some known examples in the literature.

The aim of this article is to obtain the Transmuted Exponential (TE) distribution as a special case of Transmuted Weibull distribution following the content of Aryal and Tsokos (2011) and to assess its flexibility over some other generalized models using real life data sets.

The rest of this article is organized as follows; in "The Transmuted Exponential (TE) distribution: existing and more results", the TE distribution, its properties and various statistical properties are discussed, real life applications with respect to some other wellknown generalized models shall be discussed in "Application", followed by concluding remark. The R-code for the analysis is provided as "Appendix".

\section{The Transmuted Exponential (TE) distribution: existing and more results}

A random variable $X$ is said to have a transmuted distribution function if its pdf and cdf are respectively given by;

$$
\begin{aligned}
& f(x)=g(x)[1+\lambda-2 \lambda G(x)] \\
& F(x)=(1+\lambda) G(x)-\lambda[G(x)]^{2}
\end{aligned}
$$

where; $x>0$, and $|\lambda| \leq 1$ is the transmuted parameter

$G(x)$ is the cdf of the baseline distribution.

$f(x)$ and $g(x)$ are the associated pdf of $F(x)$ and $G(x)$, respectively.

When $\lambda=0$; Eqs. (3) and (4) reduces to the baseline distribution.

If the parameter $\eta=1$ in Eqs. (4) and (5) of Aryal and Tsokos (2011), we have the pdf and the cdf of the TE distribution as;

$$
f(x)=\frac{1}{\theta} e^{-\left(\frac{x}{\theta}\right)}\left[1-\lambda+2 \lambda e^{-\left(\frac{x}{\theta}\right)}\right]
$$


and;

$$
F(x)=\left[1-e^{-\left(\frac{x}{\theta}\right)}\right]\left[1+\lambda e^{-\left(\frac{x}{\theta}\right)}\right]
$$

Respectively.

For $x>0, \theta>0,|\lambda \leq 1|$

where;

$\theta$ is the scale parameter

$\lambda$ is the transmuted parameter

\section{Special case}

For $\lambda=0$, Eq. (5) reduces to give the pdf of the Exponential distribution. Some possible plots for the pdf of the TE distribution at some selected parameter values are shown in Figs. 1, 2, 3 4, 5 and 6;

Depending on the parameter values, it can be observed from the figures above that the shape of the TE distribution could be decreasing, or inverted bathtub (unimodal). It should also be noted that $|\lambda| \leq 1$.

\section{Moments of the Transmuted Exponential distribution}

Let $\mathrm{X}$ denote a continuous random variable, the rth moment is given by;

$$
E\left(X^{r}\right)=\int_{-\infty}^{\infty} x^{r} f(x) d x
$$

Therefore, the rth moment of the TE distribution can be derived from;

$$
E\left(X^{r}\right)=\int_{-\infty}^{\infty} x^{r} \frac{1}{\theta} e^{-\left(\frac{x}{\theta}\right)}\left[1-\lambda+2 \lambda e^{-\left(\frac{x}{\theta}\right)}\right] d x
$$

This can be obtained directly from Eq. (6) of 8 when $\eta=1$ as;

$$
E\left(X^{r}\right)=\theta^{r} \Gamma(1+r)\left\{1-\lambda+\lambda 2^{-r}\right\}
$$

This can further be expressed as;

$$
E\left(X^{r}\right)=\theta^{r} r !\left\{1-\lambda+\lambda 2^{-r}\right\}
$$

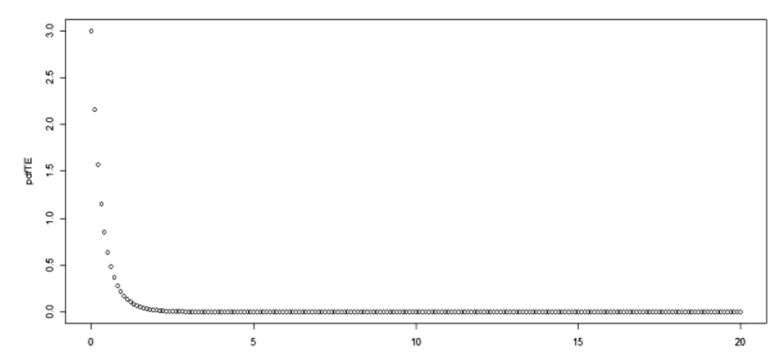

Fig. 1 Plot for the pdf of TE distribution at $(\theta=0.5, \lambda=0.5)$ 


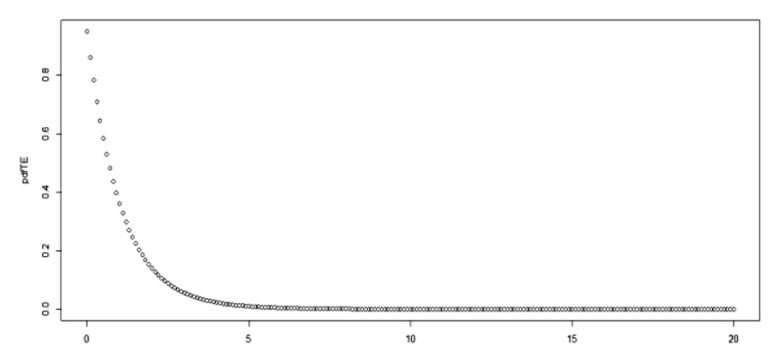

Fig. 2 Plot for the pdf of TE distribution at $(\theta=2, \lambda=0.9)$

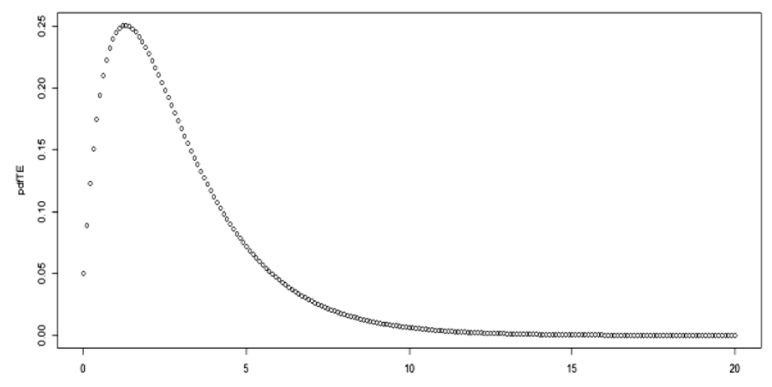

Fig. 3 Plot for the pdf of TE distribution at $(\theta=2, \lambda=-0.9)$

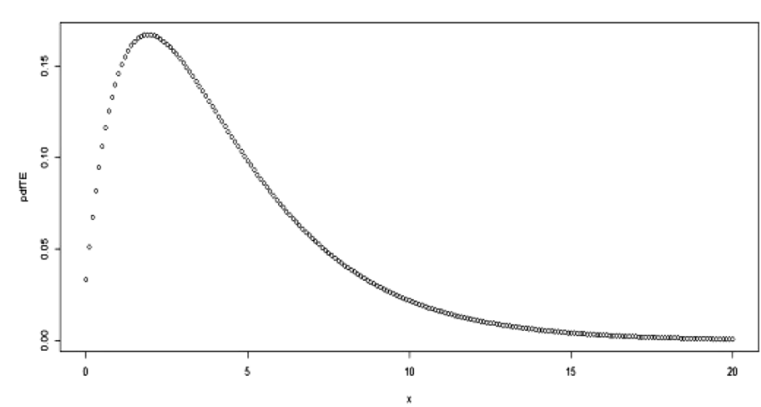

Fig. 4 Plot for the pdf of TE distribution at $(\theta=3, \lambda=-0.9)$

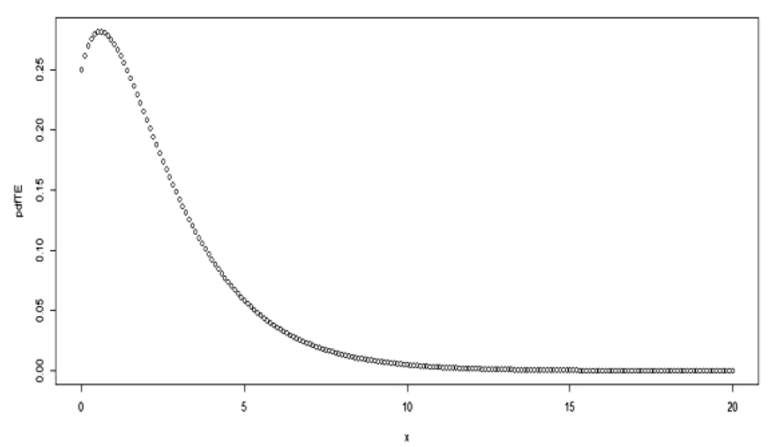

Fig. 5 Plot for the pdf of TE distribution at $(\theta=2, \lambda=-0.5)$ 


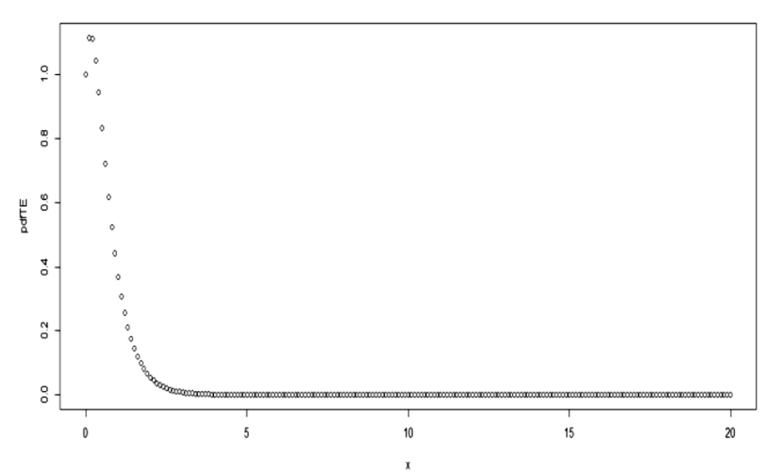

Fig. 6 Plot for the pdf of TE distribution at $(\theta=0.5, \lambda=-0.5)$

It is obvious that for $r=1$;

$$
E(X)=\theta\left(\frac{2-\lambda}{2}\right)
$$

Other higher order moments can be derived at $r>1$ from Eq. (9). The table of values (at selected values) for the mean of TE distribution is provided in Table 1.

\section{Quantile function and median of the Transmuted Exponential distribution}

The quantile function $x_{q}$ of the TE distribution can be obtained as the inverse of Eq. (6) and in particular, when $\eta=1$ in Eq. (7) of (Aryal and Tsokos (2011)) as;

$$
x_{q}=\theta\left[-\ln \left\{1-\left(\frac{1+\lambda-\sqrt{(1+\lambda)^{2}-4 \lambda q}}{2 \lambda}\right)\right\}\right]
$$

The median of the TE distribution can be obtained from Eq. (11) at $q=0.5$ as;

$$
x_{0.5}=\theta\left[-\ln \left(\frac{\lambda-1+\sqrt{1+\lambda^{2}}}{2 \lambda}\right)\right]
$$

The lower quartile and upper quartile can also be derived from Eq. (11) when $q=0.25$ and $q=0.75$ respectively.

Table 1 Table of means for the Transmuted Exponential distribution

\begin{tabular}{llllllllll}
\hline & $\lambda=-\mathbf{0 . 1}$ & $\lambda=-\mathbf{0 . 4}$ & $\boldsymbol{\lambda}=\mathbf{- 0 . 7}$ & $\boldsymbol{\lambda}=\mathbf{- 1 . 0}$ & $\boldsymbol{\lambda}=\mathbf{0}$ & $\boldsymbol{\lambda}=\mathbf{0 . 1}$ & $\boldsymbol{\lambda}=\mathbf{0 . 4}$ & $\boldsymbol{\lambda}=\mathbf{0 . 7}$ & $\boldsymbol{\lambda}=\mathbf{1 . 0}$ \\
\hline$\theta=1$ & 1.05 & 1.20 & 1.35 & 1.50 & 1.00 & 0.95 & 0.80 & 0.65 & 0.50 \\
$\theta=2$ & 2.10 & 2.40 & 2.70 & 3.00 & 2.00 & 1.90 & 1.60 & 1.30 & 1.00 \\
$\theta=3$ & 3.15 & 3.60 & 4.05 & 4.50 & 3.00 & 2.85 & 2.40 & 1.95 & 1.50 \\
$\theta=4$ & 4.20 & 4.80 & 5.40 & 6.00 & 4.00 & 3.80 & 3.20 & 2.60 & 2.00 \\
$\theta=5$ & 5.25 & 6.00 & 6.75 & 7.50 & 5.00 & 4.75 & 4.00 & 3.25 & 2.50 \\
$\theta=6$ & 6.30 & 7.20 & 8.10 & 9.00 & 6.00 & 5.70 & 4.80 & 3.90 & 3.00 \\
$\theta=7$ & 7.35 & 8.40 & 9.45 & 10.50 & 7.00 & 6.65 & 5.60 & 4.55 & 3.50 \\
$\theta=8$ & 8.40 & 9.60 & 10.80 & 12.00 & 8.00 & 7.60 & 6.40 & 5.20 & 4.00 \\
$\theta=9$ & 9.45 & 10.80 & 12.15 & 13.50 & 9.00 & 8.55 & 7.20 & 5.85 & 4.50 \\
$\theta=10$ & 10.50 & 12.00 & 13.50 & 15.00 & 10.00 & 9.50 & 8.00 & 6.50 & 5.00 \\
\hline
\end{tabular}


Random numbers from the TE distribution can be generated using the method of inversion;

$$
X=\theta\left[-\ln \left\{1-\left(\frac{1+\lambda-\sqrt{(1+\lambda)^{2}-4 \lambda u}}{2 \lambda}\right)\right\}\right]
$$

where; $u \sim U(0,1)$.

\section{Reliability analysis of the Transmuted Exponential distribution}

Mathematically, the survival function is given by;

$$
S(x)=1-F(x)
$$

Therefore, the survival function for the TE distribution can be simplified to give;

$$
S(x)=\lambda e^{-2\left(\frac{x}{\theta}\right)}-(\lambda-1) e^{-\left(\frac{x}{\theta}\right)}
$$

The hazard function is mathematically given by;

$$
h(x)=\frac{f(x)}{1-F(x)}
$$

Therefore, the expression for the hazard function (or failure rate) of the TE distribution is given by;

$$
h(x)=\frac{\frac{1}{\theta}\left[1-\lambda+2 \lambda e^{-\left(\frac{x}{\theta}\right)}\right]}{\left[\lambda e^{-\left(\frac{x}{\theta}\right)}+1-\lambda\right]}
$$

Some possible plots for the failure rate of the TE distribution at some selected parameter values are shown in Figs. 7, 8, 9 and 10;

\section{Parameter estimation and inference for the Transmuted Exponential distribution}

We make use of the method of maximum likelihood estimation (MLE) to estimate the parameters of the TE distribution. Let $X_{1}, X_{2}, \ldots, X_{n}$ be a sample of size ' $n$ ' from the TE distribution, the likelihood function is given by;

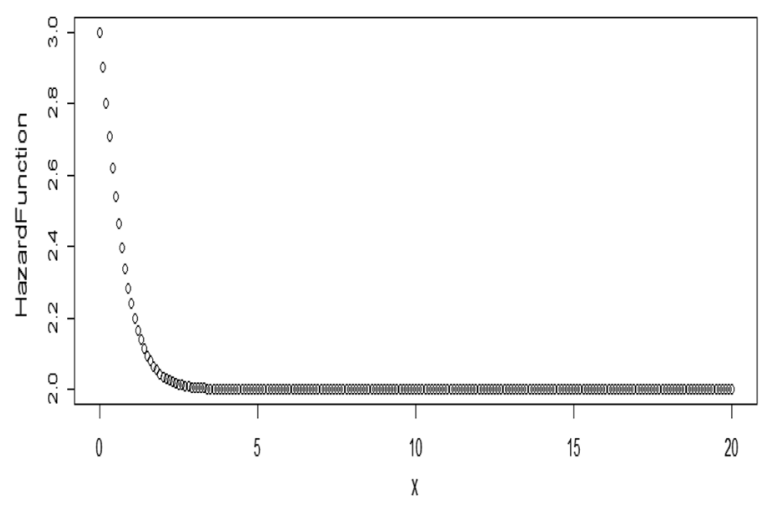

Fig. 7 Plot for the hazard function of TE distribution at $(\theta=0.5, \lambda=0.5)$ 


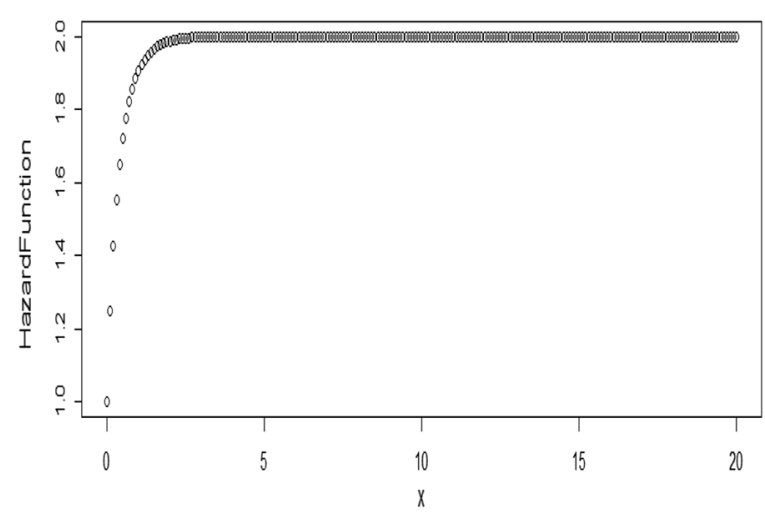

Fig. 8 Plot for the hazard function of TE distribution at $(\theta=0.5, \lambda=-0.5)$

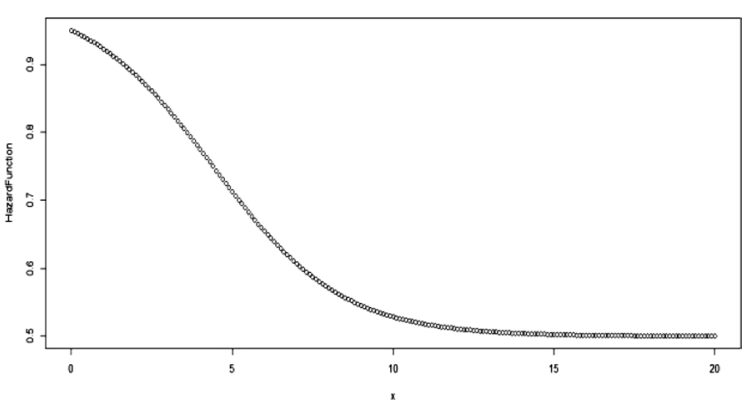

Fig. 9 Plot for the hazard function of TE distribution at $(\theta=2, \lambda=0.9)$

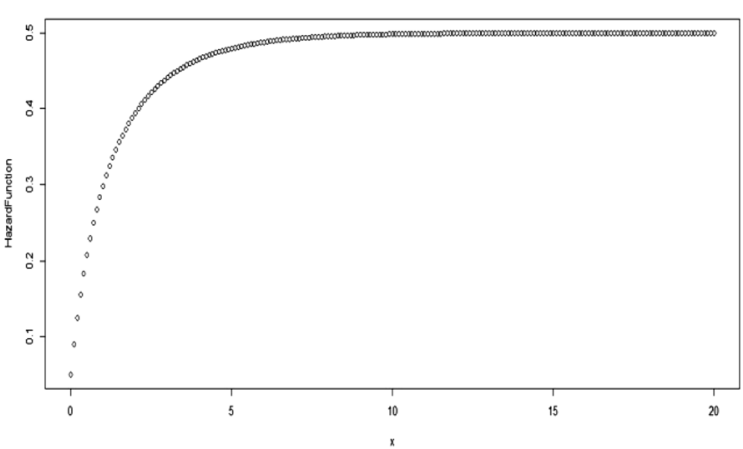

Fig. 10 Plot for the hazard function of TE distribution at $(\theta=2, \lambda=-0.9)$

$$
L\left(X_{1}, X_{2}, \ldots, X_{n} \mid \theta, \lambda\right)=\left(\frac{1}{\theta}\right)^{n} e^{-\sum_{i-1}^{n}\left(\frac{x_{i}}{\theta}\right)} \prod_{i=1}^{n}\left[1-\lambda+2 \lambda e^{-\left(\frac{x_{i}}{\theta}\right)}\right]
$$

Let $l=\log L$;

$$
l=n \log \left(\frac{1}{\theta}\right)-\sum_{i=1}^{n}\left(\frac{x_{i}}{\theta}\right)+\sum_{i=1}^{n} \log \left[1-\lambda+2 \lambda e^{-\left(\frac{x_{i}}{\theta}\right)}\right]
$$


Therefore;

$$
l=-n \log \theta-\sum_{i=1}^{n}\left(\frac{x_{i}}{\theta}\right)+\sum_{i=1}^{n} \log \left[1-\lambda+2 \lambda e^{-\left(\frac{x_{i}}{\theta}\right)}\right]
$$

Differentiating $l$ with respect to $\theta$ and $\lambda$ respectively gives;

$$
\begin{aligned}
& \frac{\partial l}{\partial \theta}=-\frac{n}{\theta} \sum_{i=1}^{n}\left[1-\left(\frac{x_{i}}{\theta}\right)\right]+\frac{2 \lambda}{\theta} \sum_{i=1}^{n} \frac{\left(\frac{x_{i}}{\theta}\right) e^{-\left(\frac{x_{i}}{\theta}\right)}}{\left[1-\lambda+2 \lambda e^{-\left(\frac{x_{i}}{\theta}\right)}\right]} \\
& \frac{\partial l}{\partial \lambda}=\sum_{i=1}^{n} \frac{2 e^{-\left(\frac{x_{i}}{\theta}\right)}-1}{\left[1-\lambda+2 \lambda e^{-\left(\frac{x_{i}}{\theta}\right)}\right]}
\end{aligned}
$$

Equating Eqs. (18) and (19) to zero and solving the resulting nonlinear system of equations gives the maximum likelihood estimates of parameters $\theta$ and $\lambda$.

We obtain the $2 \times 2$ observed information matrix through;

$$
\left(\begin{array}{l}
\hat{\theta} \\
\hat{\lambda}
\end{array}\right) \sim N\left[\left(\begin{array}{c}
\theta \\
\lambda
\end{array}\right),\left(\begin{array}{ll}
\hat{V}_{11} & \hat{V}_{12} \\
\hat{V}_{21} & \hat{V}_{22}
\end{array}\right)\right]
$$

where;

$$
V^{-1}=-E\left[\begin{array}{cc}
\frac{\partial^{2} l}{\partial \theta^{2}} & \frac{\partial^{2} l}{\partial \theta \partial \lambda} \\
\frac{\partial^{2} l}{\partial \theta \partial \lambda} & \frac{\partial^{2} l}{\partial \lambda^{2}}
\end{array}\right]
$$

The solution of the inverse matrix of the observed information matrix in Eq. (20) gives the asymptotic variance and co-variance of the maximum likelihood estimators $\hat{\theta}$ and $\hat{\lambda}$. The approximate $100(1-\alpha) \%$ asymptotic confidence interval (CI) for $\theta$ and $\lambda$ are given by;

$$
\hat{\theta} \pm Z_{\alpha / 2} \sqrt{\hat{V}_{11}} ; \quad \hat{\lambda} \pm Z_{\alpha / 2} \sqrt{\hat{V}_{22}}
$$

where; $Z_{\alpha / 2}$ is the $\alpha$-th percentile of the standard normal distribution.

\section{Application}

The models to be compared in this section include the TE distribution, Beta Exponential distribution, Generalized Exponential Distribution and the Exponentiated Exponential distribution. The analyses were performed with the aid of $\mathrm{R}$ software.

Data Set I. The first data represents the life of fatigue fracture of Kevlar 373/epoxy subjected to constant pressure at $90 \%$ stress level until all had failed. The data was extracted from (Abdul-Moniem and Seham 2015) and it has previously been used by Barlow et al. (1984). The data is as follows; 
0.0251, 0.0886, 0.0891, 0.2501, 0.3113, 0.3451, 0.4763, 0.5650, 0.5671, 0.6566, 0.6748, $0.6751,0.6753,0.7696,0.8375,0.8391,0.8425,0.8645,0.8851,0.9113,0.9120,0.9836$, $1.0483,1.0596,1.0773,1.1733,1.2570,1.2766,1.2985,1.3211,1.3503,1.3551,1.4595$, $1.4880,1.5728,1.5733,1.7083,1.7263,1.7460,1.7630,1.7746,1.8275,1.8375,1.8503$, $1.8808,1.8878,1.8881,1.9316,1.9558,2.0048,2.0408,2.0903,2.1093,2.1330,2.2100$, $2.2460,2.2878,2.3203,2.3470,2.3513,2.4951,2.5260,2.9911,3.0256,3.2678,3.4045$, 3.4846, 3.7433, 3.7455, 3.9143, 4.8073, 5.4005, 5.4435, 5.5295, 6.5541, 9.0960.

The summary of the data is provided in Table 2;

The performance of the Transmuted Exponential distribution with respect to the Beta Exponential, Generalized Exponential and Exponentiated Exponential distributions using the data on fatigue fracture is given in Table 3.

Data Set II. The second data set represents the monthly actual taxes revenue (in 1000 million Egyptian pounds) in Egypt between January 2006 and November 2010. The data was extracted from Nassar and Nada (2011). The data is as follows;

5.9, 20.4, 14.9, 16.2, 17.2, 7.8, 6.1, 9.2, 10.2, 9.6, 13.3, 8.5, 21.6, 18.5, 5.1, 6.7, 17, 8.6, 9.7, $39.2,35.7,15.7,9.7,10,4.1,36,8.5,8,9.2,26.2,21.9,16.7,21.3,35.4,14.3,8.5,10.6,19.1$, 20.5, 7.1, 7.7, 18.1, 16.5, 11.9, 7, 8.6, 12.5, 10.3, 11.2, 6.1, 8.4, 11, 11.6, 11.9, 5.2, 6.8, 8.9, $7.1,10.8$.

The summary of the data is provided in Table 4.

The performance of the Transmuted Exponential distribution with respect to the Beta Exponential distribution, Generalized Exponential distribution and the Exponentiated Exponential distribution is as shown in Table 5.

Table 2 Summary of data on fatigue fracture of Kevlar 373/epoxy at $90 \%$ stress level (to four decimal places)

\begin{tabular}{lllllllll}
\hline Min. & $\mathbf{Q}_{\mathbf{1}}$ & $\mathbf{Q}_{\mathbf{2}}$ & $\mathbf{Q}_{\mathbf{3}}$ & Mean & Max. & Variance & Skewness & Kurtosis \\
\hline 0.0251 & 0.9048 & 1.7360 & 2.2960 & 1.9590 & 9.0960 & 2.4774 & 1.9406 & 8.1608 \\
\hline
\end{tabular}

Table 3 Performance rating of selected models

\begin{tabular}{llcr}
\hline Distributions & Estimates & Log-likelihood & AIC \\
\hline Transmuted Exponential $(\theta, \lambda)$ & $\theta=1.3763, \lambda=-0.8487$ & -121.5166 & 247.0331 \\
Beta Exponential $(a, b, \theta)$ & $a=1.6797, b=1.5085, \theta=0.4849$ & -122.2275 & 250.4551 \\
Generalized Exponential $(a, \theta)$ & $a=1.70949, \theta=0.70279$ & -122.2436 & 248.4872 \\
Exponentiated Exponential $(a, \theta)$ & $a=39.969318, \theta=0.012770$ & -127.1143 & 258.2287 \\
\hline
\end{tabular}

Table 4 Summary of data on tax revenue (to two decimal places)

\begin{tabular}{lllllllll}
\hline Min. & $\mathbf{Q}_{\mathbf{1}}$ & $\mathbf{Q}_{\mathbf{2}}$ & $\mathbf{Q}_{\mathbf{3}}$ & Mean & Max. & Variance & Skewness & Kurtosis \\
\hline 4.10 & 8.45 & 10.60 & 16.85 & 13.49 & 39.20 & 64.83 & 1.57 & 5.26 \\
\hline
\end{tabular}


Table 5 Performance rating of selected models

\begin{tabular}{llll}
\hline Distributions & Estimates & Log-likelihood & AIC \\
\hline Transmuted Exponential $(\theta, \lambda)$ & $\theta=3.862 \times 10^{5}, \lambda=9.389 \times 10^{-4}$ & -83.44494 & 170.8899 \\
Beta Exponential $(a, b, \theta)$ & $a=63.52239, b=0.16957, \theta=0.76882$ & -187.9398 & 381.8795 \\
Generalized Exponential $(a, \theta)$ & $a=5.53040, \theta=0.17867$ & -191.2235 & 386.4471 \\
Exponentiated Exponential $(a, \theta)$ & $a=11.755728, \theta=0.006307$ & -212.5068 & 429.0136 \\
\hline
\end{tabular}

\section{Discussion}

The model corresponding to the lowest Akaike Information Criteria (AIC) or the highest Log-likelihood value is regarded as the 'best' model. In this case, the TE distribution has the lowest AIC value with 247.0331 and 170.8899 respectively. Also, it has the highest value of Log-likelihood of -121.5166 and -83.44494 respectively. Hence, it can be regarded as a better model for the data used.

\section{Conclusion}

This article studies the performance of the TE distribution with respect to some other generalized models. The shape of the TE distribution could be decreasing or unimodal (depending on the value of the parameters). The TE distribution appeared to be better than the Beta Exponential distribution, Generalized Exponential distribution and the Exponentiated Exponential distribution in terms of flexibility when applied two real life data. The criteria used are the Log-likelihood value and the AIC.

Authors' contributions

OPE is a research student in the Department of Mathematics, Covenant University under the supervision of Dr. AOA and Dr. EAO. He developed the idea that led to this article. The supervisors guided, read through and all agreed with the results and findings. All authors read and approved the final manuscript.

Author details

${ }^{1}$ Department of Mathematics, Covenant University, Ota, Ogun State, Nigeria. ${ }^{2}$ Department of Statistics, University of Ilorin, Ilorin, Kwara State, Nigeria.

\section{Acknowledgements}

The authors appreciate the anonymous referees for their useful and timely comments towards improving the quality of this paper. The financial support from Covenant University is also deeply appreciated.

\section{Competing interests}

The authors declare that they have no competing interests. 


\section{Appendix}

R-Code for the analysis of DATA I

FATIGUE<-

c(0.0251,0.0886, $0.0891,0.2501,0.3113,0.3451,0.4763,0.5650,0.5671,0.6566,0.6748,0.6751,0.6753,0.769$

$6,0.8375,0.8391,0.8425,0.8645,0.8851,0.9113,0.9120,0.9836,1.0483,1.0596,1.0773,1.1733,1.2570,1.276$

$6,1.2985,1.3211,1.3503,1.3551,1.4595,1.4880,1.5728,1.5733,1.7083,1.7263,1.7460,1.7630,1.7746,1.827$

$5,1.8375,1.8503,1.8808,1.8878,1.8881,1.9316,1.9558,2.0048,2.0408,2.0903,2.1093,2.1330,2.2100,2.246$

$0,2.2878,2.3203,2.3470,2.3513,2.4951,2.5260,2.9911,3.0256,3.2678,3.4045,3.4846,3.7433,3.7455,3.914$

$3,4.8073,5.4005,5.4435,5.5295,6.5541,9.0960)$

$>$ n=length(FATIGUE)

$>\mathrm{n}$

[1] 76

local(\{pkg <- select.list(sort(.packages(all.available $=$ TRUE)), graphics=TRUE)

+ if(nchar(pkg)) library(pkg, character.only=TRUE)\})

$>$ local(\{pkg <- select.list(sort(.packages(all.available $=$ TRUE)),graphics=TRUE)

+ if(nchar(pkg)) library(pkg, character.only=TRUE)\})

local(\{pkg <- select.list(sort(.packages(all.available = TRUE)), graphics=TRUE)

+ if(nchar(pkg)) library(pkg, character.only=TRUE)\})

Loading required package: miscTools

> summary(FATIGUE)

Min. 1st Qu. Median Mean 3rd Qu. Max.

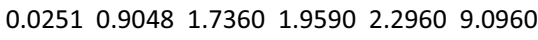

> skewness(FATIGUE)

[1] 1.940616

attr(,"method")

[1] "moment"

$>$ kurtosis(FATIGUE)

[1] 8.160792 
$>\operatorname{var}($ FATIGUE)

[1] 2.477415

loglikte<-function(p) $n * \log (1 / p[1])-\operatorname{sum}(F A T I G U E / p[1])+\operatorname{sum}(\log (1-p[2]+2 * p[2] * \exp (-(F A T I G U E / p[1]))))$

$>\mathrm{d}<-$ maxLik(loglikte, start=c(20,1))

There were 50 or more warnings (use warnings() to see the first 50)

$>$ summary(d)

Maximum Likelihood estimation

Newton-Raphson maximisation, 7 iterations

Return code 1: gradient close to zero

Log-Likelihood: -121.5166

2 free parameters

Estimates:

Estimate Std. error t value $\operatorname{Pr}(>t)$

[1,] $1.37630 .137310 .022<2 \mathrm{e}-16 * * *$

[2,] $-0.84870 .1384-6.1328 .67 \mathrm{e}-10 * * *$

Signif. codes: $0^{\prime * * * \prime} 0.001^{* * \prime} 0.01^{* \prime} 0.05$ ' $0.1^{\prime \prime \prime} 1$

$>\mathrm{AIC}(\mathrm{d})$

[1] 247.0331

loglikge<-function(p)

$n^{*} \log (p[1])+n * \log (p[2])-(p[2]) * \operatorname{sum}($ FATIGUE $)+(p[1]-1) * \operatorname{sum}(\log (1-\exp (-$ $\left.\left.\left.\left(p[2]^{*} F A T I G U E\right)\right)\right)\right)$

$>$ e<-maxLik(loglikge, start=c $(20,1))$ 
There were 50 or more warnings (use warnings() to see the first 50)

$>\operatorname{summary}(e)$

Maximum Likelihood estimation

Newton-Raphson maximisation, 7 iterations

Return code 1: gradient close to zero

Log-Likelihood: -122.2436

2 free parameters

Estimates:

Estimate Std. error $\mathrm{t}$ value $\operatorname{Pr}(>\mathrm{t})$

[1,] $1.709490 .282826 .0441 .50 \mathrm{e}-09 * * *$

$[2] \quad 0.702790 .09215 \quad ,7.6262 .41 \mathrm{e}-14 * * *$

Signif. codes: $0^{* * * \prime} 0.001^{* * \prime} 0.01^{* *} 0.05$ ' $0.1^{\prime \prime} 1$

$>\operatorname{AIC}(\mathrm{e})$

[1] 248.4872

$>\log$ likee<-function(p) $n^{*} \log (p[1])+n * \log (p[2])-\left(p[1]^{*} p[2]{ }^{*} \operatorname{sum}(\right.$ FATIGUE))

$>\mathrm{f}<-$ maxLik $(\log$ likee, $\operatorname{start}=\mathrm{c}(20,1))$

There were 50 or more warnings (use warnings() to see the first 50)

$>$ summary(f)

Maximum Likelihood estimation

Newton-Raphson maximisation, 10 iterations 


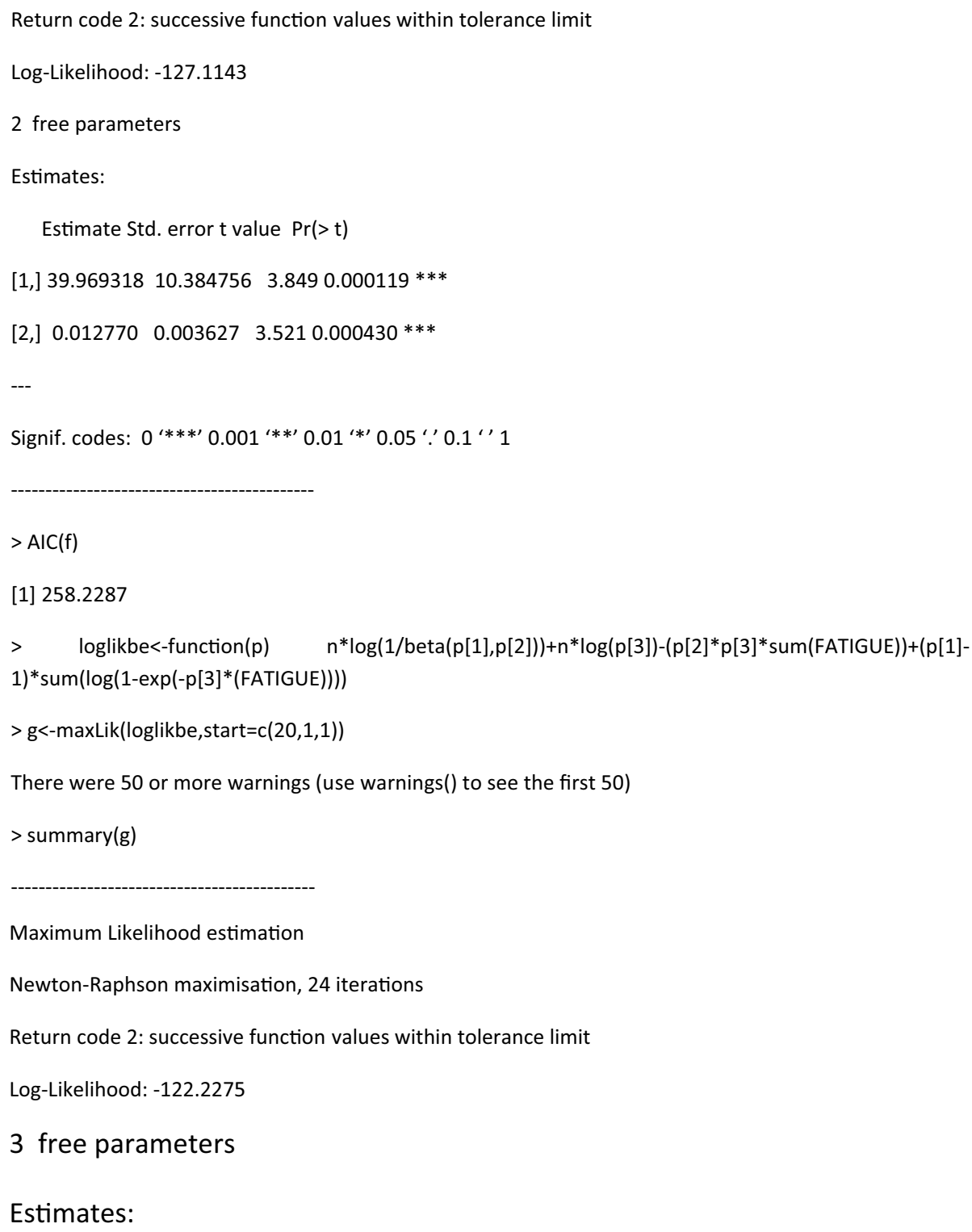


Received: 10 September 2015 Accepted: 4 December 2015

Published online: 24 December 2015

\section{References}

Abdul-Moniem IB, Seham M (2015) Transmuted Gompertz distribution. Comput Appl Math J 1(3):88-96

Afify AZ, Nofal ZM, Butt NS (2014) Transmuted complementary Weibull geometric distribution. Pak J Stat Operation Res 10(4):435-454

Ahmad A, Ahmad SP, Ahmed A (2014) Transmuted Inverse Rayleigh distribution: a generalization of the Inverse Rayleigh distribution. Math Theory Model 4(7):90-98

Ahmad K, Ahmad SP, Ahmed A (2015) On new method of estimation of parameters of transmuted size-biased exponential distribution and its structural properties. Int J Innov Res Stud 4(3):1-12

Alexander C, Cordeiro GM, Ortega EMM, Sarabia JM (2012) Generalized beta-generated distributions. Comput Stat Data Anal 56:1880-1897

Alizadeh M, Cordeiro GM, de Brito E, Demetrio CGB (2015) The beta Marshall-Olkin family of distributions. J Stat Distrib Appl 2(4):1-18

Alzaatreh A, Famoye F, Lee C (2013) Weibull-Pareto distribution and its applications. Commun Stat-Theory Methods 42(9):1673-1691

Alzaghal A, Lee C, Famoye F (2013) Exponentiated T-X family of distributions with some applications. Int J Probab Stat 2:31-49

Amini M, MirMostafaee SMTK, Ahmadi J (2012) Log-gamma-generated families of distributions. Statistics. doi:10.1008/02 331888.2012 .748775

Aryal GR, Tsokos CP (2011) Transmuted Weibull distribution: a genaralization of the Weibull probability distribution. Eur J Pure Appl Math 4:89-102

Ashour SK, Eltehiwy MA (2013a) Transmuted exponentiated modified Weibull distribution. Int J Basic Appl Sci 2(3):258-269

Ashour SK, Eltehiwy MA (2013b) Transmuted Lomax distribution. Am J Appl Math Stat 1(6):121-127

Barlow RE, Toland RH and Freeman T (1984) A Bayesian analysis of stress rupture life of Kevlar 49/epoxy spherical pressure vessels, In: Procedings. conference on applications of statistics, Marcel Dekker, New York

Bourguignon M, Silva RB, Cordeiro GM (2014) The Weibull-G family of probability distributions. J Data Sci 12:53-68

Cordeiro GM, de Castro M (2011) A New family of generalized distributions. J Stat Comput Simul 81:883-898

Cordeiro GM, Ortega EMM, da Cunha DCC (2013) The exponentiated generalized class of distribution. J Data Sci 11:1-27

Elbatal I (2013) Transmuted modified inverse Weibull Distribution: a generalization of the modified inverse Weibull probability distribution. Int J Math Arch 4(8):117-129

Elbatal I, Aryal G (2013) On the transmuted additive Weibull distribution. Aust J Stat 42(2):117-132

Eugene N, Lee C, Famoye F (2002) Beta-normal distribution and its applications. Commun Stat: Theory Methods $31: 497-512$

Gupta RD (2001) Exponentiated exponential family; an alternative to gamma and Weibull. Biometrical J 33:117-130

Gupta RD, Kundu D (1999) Generalized exponential distributions. Aust N Z J Stat 41:173-188

Gupta RD, Kundu D (2007) Generalized exponential distribution: existing methods and recent developments. J Stat Plann Inference 137:3537-3547

Hussian MA (2014) Transmuted exponentiated gamma distribution; a generalization of the exponentiated gamma probability distribution. Appl Math Sci 8(27):1297-1310

Khan MS, King R (2013) Transmuted modified Weibull distribution: a generalization of the modified Weibull probbility distribution. Eur J Pure Appl Math 6:66-88

Khan MS, King R, Hudson I (2014) Characteristics of the transmuted inverse Weibull distribution. ANZIAM J. 55(EMAC2013):C197-C217

Merovci F (2013) The transmuted rayleigh distribution. Aust J Stat 22(1):21-30

Merovci F, Puka I (2014) Transmuted pareto distribution. ProbStat Forum 07:1-11

Nadarajah S, Kotz S (2006) The Beta exponential distribution. Reliability Eng Syst Saf 91(6):689-697

Nassar MM, Nada NK (2011) The beta generalized pareto distribution. J Stat: Adv Theory Appl 6(1/2):1-17

Oguntunde PE, Adejumo AO (2015) The transmuted inverse exponential distribution. Int J Adv Stat Probab 3(1):1-7

Risti'c, Miroslav M, Balakrishnan N (2012) The gamma-exponentiated exponential distribution. J Stat Comput Simulation 82:1191-1206

Shaw WT, Buckley IR (2007) The alchemy of probability distributions: beyond Gram-Charlier expansions and a skewkurtotic-normal distribution from a rank transmutation map. Research report

Tahir MH, Cordeiro GM, Alzaatreh A, Mansoor M, Zubair M (2015) The logistic-X family of distributions and its applications. Commun Stat-Theory Methods (forthcoming)

Torabi H, Montazari NH (2012) The gamma-uniform distribution and its application. Kybernetika 48:16-30

Torabi H, Montazari NH (2014) The logistic-uniform distribution and its application. Commun Stat-Simul Comput 43:2551-2569

Zografos K, Balakrishnan N (2009) On families of beta- and generalized gamma-generated distributions and associated inference. Stat Methodol 6:344-362 